\title{
Quaderni
}

QUADERNI Communication, technologies, pouvoir

\section{La perception de l'innovation chez les chercheurs canadiens des sciences dites dures}

Innovation as perceived by Canadian researchers from so-called hard sciences

\section{Claude-Julie Bourque}

\section{(2) OpenEdition}

1 Journals

\section{Édition électronique}

URL : http://journals.openedition.org/quaderni/984

DOI : $10.4000 /$ quaderni.984

ISSN : 2105-2956

\section{Éditeur}

Les éditions de la Maison des sciences de l'Homme

\section{Édition imprimée}

Date de publication : 5 mai 2016

Pagination : 81-95

\section{Référence électronique}

Claude-Julie Bourque, « La perception de l'innovation chez les chercheurs canadiens des sciences dites dures », Quaderni [En ligne], 90 | Printemps 2016, mis en ligne le 05 mai 2018, consulté le 20 avril 2019. URL : http://journals.openedition.org/quaderni/984 ; DOI : 10.4000/quaderni.984 


\section{Dossier}

\section{La perception de l'innovation chez les chercheurs canadiens des sciences dites dures}

Au Canada, le financement public de la recherche universitaire est partagé entre le gouvernement fédéral (national) et les gouvernements régionaux des provinces et des territoires. Les fonds d'excellence canadiens et québécois sélectionnent les projets qu'ils soutiennent à travers un strict processus d'évaluation par des comités de pairs ou des comités mixtes (pairs, administrateurs, représentants du secteur privé). Les politiques scientifiques qui encadrent ces fonds s'inscrivent dans le paradigme de l'innovation introduit parallèlement à celui de l'économie du savoir depuis les années 1990. Elles se basent sur les indicateurs promus par l'Organisation de coopération et de développement économique (OCDE) selon qui la proportion des activités de recherche-développement (RD) des entreprises canadiennes serait inférieure à la moyenne de l'OCDE (OCDE $2000 ; 2005 ; 2010 ; 2014$ ).

\section{Claude-Julie Bourque}

LISST, Université Toulouse Jean-Jaurès

et CIRST, Université du Québec à Montréal
Lancée en 2007 et actualisée en 2014, la stratégie des sciences et de la technologie du Canada a pour titre Un moment à saisir pour le Canada : Aller de l'avant dans le domaine des sciences, des technologies et de l'innovation ${ }^{1}$. Le document fait référence au classement du Canada par rapport aux autres pays de l'OCDE relativement aux dépenses et aux activités en RD ainsi qu'à la production de la main-d'œuvre et il reprend la définition des quatre types d'innovation provenant du Manuel d'Oslo (OCDE, 2005). Dans l'ensemble du document, l'innovation est fortement arrimée à la recherche appliquée définie dans le glossaire comme une étude dont les résultats « visent principalement à être valides pour un seul ou un nombre restreint d'éléments, notamment des produits, des opérations, des méthodes ou des systèmes » afin de permettre de 
« donner une forme opérationnelle à des idées 》 (Ibid., p. 64). L'objectif principal de la politique est d'aider les entreprises, par des investissements gouvernementaux, à «transformer le savoir en avantages commerciaux et en nouveaux emplois » (p. 17). Les établissements d'enseignement supérieur y sont conviés à contribuer aux priorités de recherche du gouvernement ${ }^{2}$ en s'engageant dans des partenariats axés sur la recherche appliquée et en assurant la formation d'une main-d'œuvre « hautement qualifiée et compétente» (p. 24 et 61). Cette politique a fait l'objet de critiques du milieu scientifique qui y voit un assujettissement des finalités de la recherche à celles du plan d'action économique national et aux intérêts du secteur des affaires, entre autres en accordant une grande importance à la commercialisation. Néanmoins, les chercheurs s'adaptent à la nouvelle offre et aux nouveaux paramètres mis en place dans leurs demandes de subventions auprès des fonds de recherche du Canada : les instituts de recherche en santé, le Conseil de recherches en sciences naturelles et génie, le Conseil de recherches en sciences humaines, le programme des chaires de recherche et le programme des Réseaux de centres d'excellence qui relèvent ou rendent compte de leurs activités par l'intermédiaire du ministre de l'Industrie du Canada ou de Santé Canada et par d'autres institutions financées par le gouvernement canadien comme la Fondation canadienne pour l'innovation (infrastructures et équipements de pointe) ${ }^{3}$ et le Conseil national de recherche du Canada (R-D).

La nouvelle politique nationale du Québec, intitulée Priorité Emploi. Investir dans la recherche et l'innovation, c'est investir dans le Québec, ${ }^{4} \mathrm{a}$ été présentée en 2013. Le texte fait aussi allusion à la situation du Québec relativement aux pays de l'OCDE (classement avantageux des élèves québécois dans le Programme international pour le suivi des acquis des élèves, nombre de chercheurs scientifiques, investissements en recherche et en R-D (pp. $19 ; 38 ; 51-52)$ ). Ses objectifs incluent le développement des marchés " par des produits et services innovants » et l'augmentation des investissements en RD à $3 \%$ du PIB (pp. 9-10). Les innovations y sont décrites comme inspirées des découvertes ayant des retombées technologiques, sociales et organisationnelles. On y souligne l'importance du rôle des chercheurs, des « entrepreneurs scientifiques » et des « innovateurs » dans les entreprises qui sont appelés à collaborer pour promouvoir et rendre accessibles les résultats de la recherche, particulièrement aux PME. Cette politique a été initialement bien accueillie, car elle avait fait l'objet d'importantes consultations dans le milieu ${ }^{5}$ et était encadrée par le nouveau ministère de l'Enseignement supérieur, de la Recherche, de la Science et de la Technologie. Toutefois, dès son arrivée au pouvoir au printemps 2014, le nouveau gouvernement y a apporté plusieurs modifications et en a réparti la responsabilité entre le ministère de l'Éducation et de l'Enseignement supérieur et celui du Développement économique. Le milieu universitaire y a vu une atteinte au consensus et le retour à un modèle de gouvernance bicéphale et intrinsèquement contradictoire à plusieurs égards. Toutefois, les fonds de recherche conservent leur structure historique en trois axes thématiques : Nature et technologies, Société et culture, Santé.

La consultation des textes des politiques et de nombreuses réactions du milieu de la recherche dans les médias et dans différents rapports sou- 
lève la question des liens entre les discours et les pratiques. Comment les changements induits par les mécanismes opératoires découlant des politiques d'innovation influencent-ils le travail des chercheurs universitaires? En répondant à cette question, notre objectif est de décrire leurs perceptions des incitations au changement et de leurs impacts sur la qualité de la recherche et de la formation à la recherche. Pour ce faire, nous utilisons les données d'une enquête empirique dont nous présentons la littérature de référence, le cadre notionnel et les méthodes. Les résultats des analyses sont ensuite présentés et discutés.

\section{Les enjeux de l'innovation}

Les critiques du paradigme de l'innovation portent souvent sur la validité et les fondements idéologiques des notions et des modèles axés sur des principes normatifs et directifs qui promeuvent des transformations des pratiques universitaires visant à arrimer étroitement le système scientifique aux valeurs et aux dynamiques du marché des affaires (Godin, 2006 ; Milot, 2003). On soulève aussi la menace potentielle que ces modèles font peser sur la culture scientifique, l'éthique publique et l'autonomie du champ universitaire (Bernatchez, 2010, Chan et Fisher, 2006 ; Freitag, 1995) ainsi que les risques de déséquilibre qu'ils engendrent en avantageant les objets commercialisables et la Big Science (Giroux, 2006).

Trois études empiriques sur les sciences dites dures (sciences de la santé, de la nature et génie ${ }^{6}$ ) ont inspiré nos questionnements. La première traite de l'ouverture des chercheurs états-uniens en sciences de la vie à la collaboration avec les milieux non universitaires et leurs réserves à l'égard de la commercialisation (Owen-Smith et Powell, 2004). Dans la première phase de notre recherche, nous avons corroboré certains résultats de cette enquête en démontrant que la majorité des chercheurs sont défavorables à la commercialisation qu'ils considèrent comme une menace à la liberté académique, alors qu'une majorité plus importante ne s'oppose pas au chevauchement interinstitutionnel à l'endroit duquel ils adoptent des postures plutôt pragmatiques (Bourque, 2011a).

Selon une autre enquête étatsunienne sur le processus du choix des problèmes de recherche en biologie, la commercialisation est un phénomène médiatisé par certains chercheurs à travers des rapports diversifiés à une culture scientifique dont les principes fondamentaux semblent stables malgré des changements notables dans les pratiques (Cooper, 2009). Ces résultats convergent avec nos travaux qui montrent que la collaboration directe avec les entrepreneurs est un phénomène très marginal qui se limite généralement aux questions relatives au financement et non à celles portant sur le choix des objets et des méthodes ou sur la diffusion des résultats. Ceci est vrai même dans un domaine comme celui de la biotechnologie où les enjeux liés à la propriété intellectuelle et à la commercialisation sont très importants (Bourque, 2011a ; 2011b).

Enfin, une enquête dans le domaine des nanosciences et nanotechnologies en France rend compte des stratégies utilisées pour faire face aux « injonctions de performance », de la construction de sens par les chercheurs devant les « nouveaux horizons normatifs » et de leur rapport parfois 
difficile aux mécanismes des programmes de financement souvent marqué par la confusion et l'incertitude (Hubert, Chateauraynaud et Fourniau, 2012).

Les résultats de ces études mettent en cause l'adaptation des pratiques de recherche aux valeurs portées par la conception managériale et programmatique de la science, notamment le déplacement des mécanismes d'allocation des ressources des pairs aux experts et à de nouvelles formes de régulation des activités par une forte action bureaucratique et évaluative (Barrier, 2011 ; Vilkas, 2009). Cette action se caractérise entre autres par des indicateurs d'excellence et de performance qui seraient utilisés de manière inadéquate (pour évaluer les individus plutôt que les grandes tendances des systèmes et des disciplines), selon Gingras (2014). Ce dernier souligne d'ailleurs l'absence de fondements épistémologiques de ces procédures et leur pertinence, joignant sa voix à d'autres critiques des évaluations «quantophréniques » du New Public Management qui serait atteint par la «maladie de la mesure » (de Gaulejac, 2012).

\section{Cadre notionnel et méthodologique}

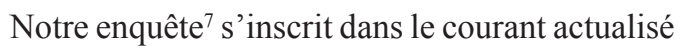
des études de champ anglo-saxonnes (Higher Education, sociologie des sciences) (Burri, 2008; Cooper, 2009 ; Gonzales, 2014), donc certains des principaux concepts de la sociologie de la pratique ont été mobilisés afin de saisir les enjeux de pouvoir à l'échelle structurale autant que les pratiques collectives et individuelles (Bourdieu, 2001 ; 1984 ; 1976; Bourdieu et Wacquant, 1992). Nous avons produit des données sur la perception de l'entrecroisement des structures objectives des champs universitaire, économique et politique dans les dynamiques scientifiques, sur les enjeux liés à la compétition pour l'accès aux ressources, sur les différentes formes de capital mobilisées (économique, scientifique, social, symbolique), ainsi que sur les croyances, le sens et la valeur qui animent, définissent et caractérisent le champ scientifique à l'aide des principaux concepts de la théorie (habitus, illusio, doxa). Cette approche compréhensive a permis de mettre en lumière les tensions médiatisées par les agents entre les forces structurelles qui les contraignent, les forces intériorisées qui se manifestent dans leurs pratiques ainsi que la marge de manœuvre dont ils disposent et, conséquemment, des risques que représentent les changements qu'ils adoptent (ou rejettent) pour le maintien de l'intégrité et de l'autonomie du champ.

Le dispositif de l'enquête, de nature mixte et longitudinale, comprend un sondage en ligne (questions ouvertes et fermées), l'archivage de curriculum vitæ, des entretiens en profondeur ainsi que des observations participantes ${ }^{8}$. Le sondage, tenu en 2009-2010, comprend les réponses de 735 chercheurs présents dans six grands groupes universitaires ${ }^{9}$ au Québec, et affiliés aux secteurs des sciences de la santé (SS) $(43,7 \%)$, des sciences naturelles ( $\mathrm{SN})(38,2 \%)$ et du génie (SG) $(18,2 \%)$ dans 86 disciplines (surtout la biologie, la chimie et la biologie cellulaire). Ils ont amorcé leur carrière en recherche entre 1958 et 2009 ( $57 \%$ avant 1995$)$ et les femmes représentent $23 \%$ de l'échantillon. Les 73 chercheurs en formation sont des doctorants et des chercheurs postdoctoraux ( $41 \%$ de femmes). La campagne d'entretiens se poursuit depuis le printemps 2010 
avec des chercheurs et des administrateurs invités à commenter les résultats des sondages et les actualités universitaires. Les observations participantes ont lieu depuis l'hiver 2009 lors de conférences, de séminaires et d'ateliers portant sur les résultats préliminaires de l'enquête. Les analyses qualitatives pour cet article sont réalisées sur le corpus de données textuelles $(\mathrm{N}=832)$ qui ont été traitées avec le logiciel NVivo ${ }^{10}$.

\section{Résultats}

Une analyse quantitative simple des données sur les opinions (échelles de Likert) permet de constater qu'une majorité des chercheurs s'opposent à la commercialisation, à l'intégration de la contribution à l'économie dans les missions fondamentales de l'université, et surtout à l'utilisation des retombées pratiques et de la valeur économique des découvertes comme critères d'évaluation de la qualité de leur travail. Par contre, ils sont plutôt favorables en principe aux collaborations externes et à l'entrepreneuriat universitaire (entreprises, brevets, licences).
Dans cet article, les principaux résultats présentés sont de nature qualitative, car ils permettent de répondre avec plus de profondeur à notre question. La figure suivante présente les principaux thèmes repérés dans les réponses à des questions ouvertes ${ }^{11}$ dans lesquelles l'innovation est abordée positivement et/ou négativement par les répondants relativement au thème en question. L'analyse des extraits associés à ces thèmes rend compte des effets d'usage paradoxaux de la notion d'innovation sur le plan interprétatif comme sur celui des pratiques.

\section{Le sens des mots}

S'agissant de la définition de l'innovation, nous avons constaté que bien qu'ils en maîtrisent les cadres opératoires dans les paramètres des politiques liées au financement et à l'évaluation de la recherche, les chercheurs donnent spontanément divers sens au terme. Dans les opinions exprimées sur le changement influençant la qualité de la recherche et de la formation, nous distinguons trois axes interprétatifs de l'innovation : 1) les

\begin{tabular}{|c|c|c|c|c|}
\hline Thèmes & $\mathbf{N}$ & Sous-thèmes & Perception positive & Perception négative \\
\hline Financement & 661 & $\begin{array}{l}\text { accessibilité, sources, } \\
\text { évaluation et compétition, } \\
\text { ressources humaines }\end{array}$ & ++ & --- \\
\hline $\begin{array}{l}\text { Approche } \\
\text { scientifique }\end{array}$ & 515 & $\begin{array}{l}\text { stratégie et rapport au risque, } \\
\text { créativité, liberté académique, } \\
\text { autonomie, collaboration, } \\
\text { multidisciplinarité }\end{array}$ & ++ & -- \\
\hline Charge de travail & 392 & & & -- \\
\hline $\begin{array}{l}\text { Progrès techniques } \\
\text { et technologiques }\end{array}$ & 388 & $\begin{array}{l}\text { Données, techniques, } \\
\text { équipements }\end{array}$ & ++ & - \\
\hline Formation & 211 & & + & - \\
\hline
\end{tabular}


infrastructures et équipements auxquels ils ont accès ; 2) la quête de nouvelles idées qui marque leurs démarches ; 3 ) les dispositifs économiques et managériaux qui les contraignent.

Le terme revient souvent dans des commentaires enthousiastes lorsqu'il est question des innovations technologiques (internet, équipements de pointe, outils et méthodes, bases de données, etc.) ainsi que des progrès récents de la science fondamentale (génomique, protéomique, bioinformatique, etc.). Les propos sont élogieux à l'endroit de Fondation canadienne pour l'innovation (FCI) (surtout chez des chercheurs en SN déjà en poste avant sa création en 1997). Plusieurs répondants considèrent que la fondation a permis l'acquisition et l'amélioration d'infrastructures majeures (installations et équipements de pointe), un net avantage pour les grands groupes comme les réseaux d'excellence et pour les jeunes chercheurs. Ces investissements auraient selon certains « changé positivement la dynamique de développement de la recherche » et en auraient amélioré la portée internationale. Toutefois, des réserves sont aussi exprimées (surtout en SS) sur la taille des réseaux financés qui mène au fractionnement des montants alloués entre des dizaines d'équipes et de projets. On note aussi qu'une grande part des montants «va dans $d u$ béton » et qu'il reste difficile de financer l'achat de « matériel de prix intermédiaire » qui est moins spectaculaire, mais tout aussi nécessaire que les grands équipements ${ }^{12}$ : «Ça paraît bien, mais le montant n'est pas utilisé pour créer ». L'instabilité chronique du financement des étudiants et du personnel de recherche conduirait par ailleurs à la perte d'expertise et de compétences, considérée par les chercheurs comme une entrave à l'utilisation maximale et à la pérennité de ces ressources : "Il faut se rappeler que ce sont les personnes qui font la recherche et qui développent les nouvelles idées. Il faut investir dans les personnes ».

Notre deuxième constat porte sur l'importance dans le discours des répondants de « l'esprit d'innovation » qui marquerait l'approche scientifique du milieu universitaire canadien, en particulier dans les expériences de collaboration entre les chercheurs de diverses disciplines qui travaillent moins « en vase clos » qu'auparavant. La qualité du travail accompli avec les bureaux de liaison entreprise-université ainsi qu'avec les services de valorisation et de gestion de la propriété intellectuelle est soulignée et explique en partie l'ouverture aux collaborations externes. Qu'ils soient engagés ou non dans des projets collaboratifs, les chercheurs sont bien informés et expriment de l'intérêt envers les besoins de la société, les développements industriels, le travail en clinique, etc.

Les chercheurs en SG se sentent plus à l'aise avec l'orientation pratique des partenariats d'affaires. Toutefois, en SN et SS, plusieurs estiment que les dispositifs qui imposent l'engagement dans la recherche appliquée et commercialisable reflètent un « modèle unique » qui ne convient pas à toutes les disciplines ni à tous les objets de recherche. Ils parlent avec passion de projets audacieux dont ils rêvent, mais qui ne peuvent s'inscrire dans les paramètres étroits qui leur sont imposés et regrettent devoir concentrer leurs efforts sur des projets fortement balisés par les acquis et les besoins sociétaux : «Ça ne coûte pas cher, on est pas mal certain que ça va 
fonctionner ». Le contexte permettant de créer une véritable innovation scientifique nécessiterait deux intrants essentiels : une idée vraiment originale portée par un chercheur « visionnaire » et « des fonds qui vont durer pour un bon effort de cinq à dix ans ». Les commentaires sur le temps nécessaire à la production scientifique reviennent très souvent dans les critiques des chercheurs qui se désolent de la superficialité des travaux axés sur les retombées immédiates «aux dépens de la découverte de nouveaux paradigmes porteurs et de nouvelles avenues de recherche ». Plusieurs chercheurs témoignent aussi de la dispersion du temps à laquelle ils font face, car le rythme de production doit constamment être réorganisé en fonction de la multiplication exponentielle de leurs tâches, surtout chez ceux qui ont une fonction professorale :

"Le type de travail au quotidien a beaucoup changé ; nous passons notre temps à écrire des demandes de subventions au lieu de lire, prendre le temps de développer des idées originales et même discuter avec les étudiants (...) les organismes exigent de plus en plus de résultats préliminaires convaincants avant d'obtenir l'argent. Donc, au bilan, il faut avoir fait le travail avant même d'avoir l'argent pour le faire. (...) Ce besoin de savoir à l'avance ce que l'on doit obtenir me semble contradictoire avec la mission même de la recherche.»

Les finalités purement scientifiques de la recherche sont défendues par plusieurs chercheurs qui ont l'impression que la recherche fondamentale est évacuée dans le paradigme de l'innovation. Certains commentent le fait que la créativité leur semble «plus restreinte aux aspects tech- niques » et d'autres jugent que «la transition vers la recherche appliquée a un peu détruit le côté "aventurier" de la recherche en orientant tout vers la compétition et la performance à court terme, ce qui n'est pas compatible avec les développements scientifiques les plus intéressants ». Le manque de financement "pour les recherches innovatrices d'envergure modérée " et l'élitisme des chercheurs bien établis dans les grands réseaux sont des problèmes très souvent soulignés : «Des taux de succès d'à peine $20 \%$ ont un effet très inhibiteur sur l'élaboration de projets un peu risqués, qui ne sont pas certains de donner les résultats prévus. Or, sans imprévu, où est l'innovation? »

Des chercheurs et des administrateurs critiquent aussi les ruptures et le «manque de pérennité et de cohérence " provoqué par les changements brusques dans la gouvernance, les politiques et les programmes scientifiques auxquels ils doivent constamment s'ajuster : « il y a une tendance des organismes à donner plus d'appels d'offres sur des sujets et thématiques ciblés. Le but est de faire $d u$ "top down" et de s'assurer que l'investissement de fonds publics en recherche rapporte. " Le souci du «retour sur l'investissement » et de la productivité transparaît pour plusieurs dans le discours public et médiatique des institutions : «Dans les journaux, je vois beaucoup d'articles de l'université $[X]$. Une innovation ici, une innovation là.... c'est bien, mais je pense qu'ils exagèrent $»$.

Les institutions de recherche étant elles-mêmes soumises à des évaluations très strictes, elles ne se préoccupent pas que de leur image, mais mettent aussi en place de nombreuses procé- 
dures de contrôle pour répondre aux exigences de transparence et de reddition de comptes (qui sont explicites dans la politique scientifique canadienne d'ailleurs). Ces tâches souvent aliénantes et chronophages détournent les chercheurs de leurs activités de recherche et d'enseignement et ils expriment de l'exaspération à l'égard de «la bureaucratie croissante qui ralentit tout le système ». Ces procédures alourdiraient une charge déjà très importante, ce qui «ne laisse finalement pas tant de temps à la recherche, qui s'exécute principalement par les étudiants [car] il n'y a généralement pas assez de fonds pour engager des techniciens ou assistants de recherche ».

\section{Le sens des actions}

Relativement aux pratiques, nous avons remarqué des tensions provoquées par l'interprétation et l'appropriation des dimensions opératoires du paradigme de l'innovation particulièrement dans trois types d'activités : la collaboration, la formation doctorale et les stratégies programmatiques. Les chercheurs ont des opinions contrastées à l'égard des pratiques de collaboration fortement promues dans les politiques scientifiques au sujet desquelles les points de vue diffèrent selon l'expérience et le secteur de recherche. Ils apprécient les interactions avec leurs collègues d'autres laboratoires et disciplines, notamment dans le contexte des réseaux thématiques qui leur donnent accès à des ressources importantes (intellectuelles et technologiques), et dans celui de l'interdisciplinarité qui leur permet de sortir du « travail en silo ». La mixité disciplinaire et organisationnelle soulève des résistances chez ceux qui la perçoivent comme un modèle que l'on cherche à leur imposer.
En $\mathrm{SG}$, ce modèle semble mieux convenir à la culture disciplinaire historiquement plus ancrée dans les partenariats industriels et la RD en Amérique du Nord. Cependant, en SS et SN, ils sont nombreux à souligner que cette injonction peut mener à des regroupements artificiels, à des lourdeurs administratives et à des luttes internes nuisibles. Ils sont aussi souvent confrontés à des difficultés relatives à l'évaluation (des individus et des projets) et à la publication de travaux multidisciplinaires : "on ne forme pas nos étudiants encore de cette façon-là. (...) Pour avoir une promotion à l'intérieur de ton département, il faut que tu sois assez "monodisciplinaire" ».

Deuxièmement, la formation de la relève scientifique est marquée par des discours qui se déploient dans deux directions. D'une part, les chercheurs expriment leur désir d'améliorer et de mieux adapter la formation aux changements dans les pratiques et au marché de l'emploi scientifique en ajoutant plus de formation fondamentale (jugée essentielle pour le travail interdisciplinaire), plus de réflexivité, une maîtrise plus large et plus profonde des savoirs et des savoir-faire et plus de formations d'appoint en fonction des types de carrière visés (communication, gestion de projet, analyse des impacts sociaux, etc.).

D'autre part, la majorité des chercheurs expriment de l'inquiétude à l'égard de la volonté de réduire la durée de la formation doctorale et jugent qu'une formation trop rapide serait nuisible à la réputation institutionnelle. En SN et SS surtout, on s'interroge sur l'origine de cette idée et sur la nature des données sur lesquelles elle se fonde. Certains soulignent que c'est au cœur d'une formation fondamentale de haut niveau 
que se forge la réelle capacité d'innover et qu'il faut au minimum de quatre à cinq ans après une solide formation préparatoire au deuxième cycle pour former de véritables chercheurs et non des « super techniciens ». En SG toutefois, ils sont remarquablement plus nombreux à favoriser une formation ne dépassant pas trois ans, réalisée surtout en milieu de pratique et axée sur la gestion de projet en recherche appliquée et en RD.

Enfin, plusieurs chercheurs s'expriment sur leurs pratiques d'adaptation et de planification. Ils savent « décoder » les paramètres et concevoir des stratégies pour accéder aux ressources dont ils ont besoin dans un contexte marqué par une « compétition féroce ». Néanmoins, il existe à cet égard une forte tension entre l'interprétation de la notion d'innovation telle qu'elle est développée dans les politiques et les programmes et le sens que les chercheurs lui attribuent. En effet, pour l'avancement de leur carrière et pour le financement de leurs projets, l'adoption de certaines postures stratégiques est décrite par des commentaires parfois cyniques sur ce qu'ils doivent faire pour passer les barrières des évaluations, y compris dans les dynamiques internes du système universitaire pour certains qui jugent que « le Cheval de Troie de l'économisme » est entré dans les institutions.

Ainsi, plusieurs d'entre eux disent éviter de se lancer dans des voies peu explorées pour tenter d'expliquer des phénomènes nouveaux en renonçant à proposer des projets qu'ils estiment être les plus intéressants scientifiquement, mais qui sont « un peu plus dangereux ». Ils s'arment donc de patience et remettent ces «meilleures idées » à plus tard en espérant acquérir la notoriété leur permettant plus de liberté après quinze ou vingt ans et relaient cette idée à leurs jeunes collègues : « Malheureusement, parfois on a des gens qui veulent innover de façon incroyable. Mais ce n'est vraiment pas le temps de faire ça! (...) Au début, on connaît la règle, il faut faire des trucs simples. " Ils investissent aussi beaucoup de temps à produire des « prérésultats » et à formuler soigneusement des projets dans lesquels ils vont « suivre la mode et s'en tenir à ce qui marche ». L'un d'eux exprime laconiquement la situation paradoxale à laquelle il doit faire face : « il faut vraiment arriver à dire que c'est nouveau, mais pas trop nouveau ».

\section{Discussion et conclusion}

En analysant un vaste corpus de données provenant d'un échantillon représentatif de la variété des disciplines des sciences dites dures, nous avons produit des résultats ancrés empiriquement et basés sur la répétition de dizaines de témoignages concordants qui rendent les descriptions produites irréductibles à l'anecdotique et utiles pour la mise en relation avec d'autres études afin d'en enrichir la portée internationale et de contribuer au cumul et à la consolidation des savoirs sur le changement dans le champ scientifique.

Les dimensions interprétatives dégagées dans notre analyse ont permis de mettre en exergue des discours qui s'expriment de manière plus radicale au sujet des visées économiques et plus pragmatiques lorsqu'il s'agit des dispositifs, comme l'ont fait aussi Hubert et ses collaborateurs (2012) en France. Ces discours s'articulent autour de l'accès aux ressources essentielles à la recherche, en particulier les infrastructures 
et technologies de pointe, et sur les stratégies complexes des agents les plus fortement dotés en capital. On remarque d'ailleurs la centralité des enjeux liés à l'adhésion à de grands collectifs, lieux de concentration du capital scientifique, social et symbolique constitutif de leur identité et facilitant leur mobilité dans le champ comme cela a déjà été démontré (Burri, 2008 ; Cooper, 2009). En général, ils sont aussi très ouverts au principe des collaborations interdisciplinaires ou interinstitutionnelles, bien qu'ils critiquent la tendance souvent contre-productive à vouloir généraliser ces pratiques.

Il s'agit là d'un résultat original important, car il met en lumière la contrainte de l'adaptation à de nouvelles normes d'évaluation de la pertinence des thèmes et de la qualité des projets scientifiques. Ces normes sont historiquement un objet de lutte pour la constitution et la reproduction du champ scientifique bien sûr, toutefois les chercheurs ont la nette impression que le contrôle de la définition de la qualité de leur production leur échappe de plus en plus, et tout particulièrement les critères et les processus servant à l'évaluer. Ils réaménagent donc la hiérarchie des priorités de leurs projets dont ils redéfinissent l'échéancier en fonction de nombreuses mesures évaluatives. Paradoxalement, leur engagement dans cette forme de compétition contribue à la perte de l'autonomie du champ scientifique (national) et à la transformation de leurs propres institutions en « striving universities », qui sont aussi engagées dans une compétition axée sur la productivité, la visibilité et le prestige (Gonzalez, 2014).

Cette dynamique caractérisée par l'urgence subordonne le travail des chercheurs à des diktats managériaux institutionnels perçus comme un système d'encadrement et de surveillance qui les aliène, entre autres par la multiplication exponentielle de tâches cléricales pour lesquelles ils sont nettement surqualifiés alors même que le soutien de ressources humaines de proximité (dans les départements et les laboratoires) est réduit au strict minimum. Ceci a un impact sur la représentation qu'ont les chercheurs des doctorants et des chercheurs postdoctoraux qu'ils supervisent et qu'ils désignent spontanément comme du «personnel de laboratoire » jugé crucial pour assurer la cadence de la production incluant les publications. Tout se passe comme si aucune instance n'évaluait le cumul de tâches qui réduit le temps disponible pour la recherche et l'enseignement ni les coûts réels ou l'efficacité de tous ces contrôles évaluatifs. Cette dynamique reflète une modification concrète des pratiques institutionnelles et individuelles qui sont ajustées aux conditions de financement appuyées sur quelques dimensions strictement quantitatives (Barrier, 2011 ; de Gaulejac, 2012). Cette situation est, comme l'affirme Gingras (2014), tout à fait contraire à la logique selon laquelle les modes d'évaluation devraient être conçus et développés en fonction des pratiques sur lesquelles ils portent.

L'enjeu principal lié à l'idéologie de l'innovation nous semble résider dans le processus de reconstruction de l'habitus et de l'illusio scientifiques qui président aux valeurs, aux pratiques et aux règles de légitimation du champ (Bourdieu, 2001 ; Bourdieu et Wackant, 1992). Ces changements résultent de pressions externes qui sont souvent médiatisées par les chercheurs eux-mêmes, comme l'ont constaté de Cooper (2009) aux États-Unis ainsi que Hubert et ses collaborateurs (2012) en 
France, entre autres par la «frilosité » qui caractérise les stratégies d'évitement des risques. Les dynamiques parallèles de soumission et de résistance aux changements dans les règles du jeu pour l'accès aux ressources provoquent ainsi une crise de la doxa révélée dans d'autres études récentes qui mettent en exergue la contreproductivité des tensions entre les cadres construits par la profession et « la capacité des chercheurs à gérer de multiples contraintes externes en situation de travail » (Barrier, 2011, p. 534).

Ces comparaisons entre nos résultats et ceux d'autres études récentes dans d'autres pays rendent compte de la pertinence de l'approche structuro-constructiviste comme cadre conceptuel pour la production et l'analyse des données (Bourdieu, 2001). Afin de dépasser les limites de cette analyse, il serait pertinent d'utiliser le même modèle pour produire des données comparatives en incluant les disciplines des sciences humaines et sociales. Il serait aussi intéressant d'intégrer aux analyses en cours d'autres approches comme celle des critiques contemporaines de la sociologie des professions sur les enjeux politiques au sein des groupes autonomes (Champy, 2012), ou encore le modèle des systèmes d'innovation, jamais remis en cause par les chercheurs dans notre échantillon (Niosi, 2014).

Nous concluons en insistant sur le fait que les chercheurs ne s'opposent pas à ce que les gouvernements stimulent des innovations porteuses de retombées économiques, mais plutôt à ce que ce modèle s'impose à l'ensemble du champ universitaire. En effet, les prémisses des politiques scientifiques qui viennent de l'OCDE concernent la productivité des entreprises en
R-D (hauteur des investissements et qualité de la main-d'œuvre) et non la nature ou la qualité de la recherche universitaire pourtant intégrée à la rhétorique du changement et de l'innovation. Cette distinction est claire dans le discours des chercheurs pour qui la notion d'innovation a un sens axé sur son potentiel perturbateur et révolutionnaire plus près de Schumpeter dans sa dimension socio-économique et de Kuhn dans sa dimension paradigmatique. Leur habitus est par ailleurs caractéristique d'une vision profondément bachelardienne fondée sur le renouvellement des questions scientifiques, la problématisation et la conceptualisation. Les paradoxes qui marquent leurs pratiques vont donc au-delà du financement et de la pondération de priorités institutionnelles (Owen-Smith et Powell, 2004) et les effets indésirables du discours se répercutent aussi dans les disciplines des sciences dites dures et de la Big Science (Giroux, 2006) dont la dimension fondamentale est aussi mise en cause.

Les effets d'usage paradoxaux associés à la notion d'innovation marquent les efforts de protection de l'identité professionnelle des scientifiques et de l'autonomie de leur champ de pratique. Si l'usage même du terme innovation était déjà perçu comme une forme de ruse des " dominés du champ scientifique » dans leur quête de légitimité au milieu des années 1970 (Bourdieu, 1976, p.104), il semble maintenant aussi être lié à la lutte de pouvoir entre les champs scientifique, économique et politique. Plus précisément, les tentatives d'effacement des frontières entre ces champs mettent en cause les logiques politiques, managériales et comptables souvent incompatibles avec la production de connaissances qui reste pour la majorité des chercheurs 
l'objectif ultime de leurs travaux. En effet, bien qu'ils ne les considèrent pas systématiquement incompatibles, les chercheurs universitaires établissent une distinction nette entre la quête de vérité de la recherche scientifique (fondamentale et appliquée) de celle de profitabilité, de la R-D (commerciale et industrielle).

\section{$R \cdot E \cdot F \cdot E ́ R \cdot E \cdot N \cdot C \cdot E \cdot S$}

C. BARRIER, « La science en projets : financements sur projet, autonomie professionnelle et transformations du travail des chercheurs académiques », in Sociologie du Travail, n53 (4), 2011, pp. 515-536.

J. BERNATCHEZ, « La valorisation commerciale de la recherche universitaire. Principes, modalités et enjeux d'éthique publique », in Éthique publique, $\mathrm{n}^{\circ}$ 12(1), 2010, pp. 55-78.

P. BOURDIEU, Science de la science et réflexivité, Paris, Raisons d'Agir, 2001.

P. BOURDIEU, Homo Academicus, Paris, Minuit, 1984.

P. BOURDIEU, Le champ scientifique, in Actes de la recherche en sciences sociales, $\mathrm{n}^{\circ} 2(2)$. 1976, pp. 88-104.

P. BOURDIEU et L. WACQUANT, An Invitation to Reflexive Sociology, Chicago : University of Chicago Press, 1992.

C.J. BOURQUE, Le changement comme tradition dans la recherche et la formation à la recherche en biotechnologie et en périphérie : étude de cas en sciences de la santé, sciences naturelles et génie, Thèse de doctorat en éducation, Canada, Université de Sherbrooke, 2011(a).

C.J. BOURQUE, « Introduction du générateur de liens sociaux par contexte (GLSC) dans une approche mixte : étude de l'hétérogénéité dans les dynamiques sociales des chercheurs en biotechnologie et sciences de la vie, in Bulletin de Méthodologie Sociologique, ${ }^{\circ}$ 110(1), pp. 58-73. R.V. BURRI, « Doing Distinctions, Boundary Work and Symbolic Capital in Radiology », in Social studies of Science, $\mathrm{n}^{\circ} 38(1), 2008$, pp. 35-62. 
F. CHAMPY, La sociologie des professions, Paris, Presses universitaires de France, 2012.

A.S. CHAN et D. FISHER, "Academic Culture in Canadian Universities: The Contexts of Change", in Y. Gingras \& L. Roy (Eds.), Les transformations des universités du XIII au XXI siècle, Québec, Presses de l'Université du Québec, 2006, pp. 175-203.

M.H. COOPER, "Commercialization of the University and Problem Choice by Academic Biological Scientists" in Science, Technology \& Human Values, no 34(5), 2009, pp. 629-653. $\mathrm{V}$. DE GAULEJAC, La recherche malade $d u$ management, Versailles, Éditions Quae, 2012.

M. FREITAG, Le naufrage de l'université, Paris, Découverte, 1995.

A. GIROUX, Le pacte faustien de l'université, Montréal, Liber, 2006.

Y. GINGRAS, Les dérives de l'évaluation de la recherche. Du bon usage de la bibliométrie, Paris, Raisons d'agir, 2014.

L.D. GONZALES, "Framing Faculty Agency Inside Striving Universities: An application of Bourdieu's theory of Practice", Journal of Higher Education, no 85 (2), 2014, pp. 193-218.

B. GODIN, "The Knowledge-based Economy: Conceptual Framework or Buzzword? in Journal of Technology Transfer, n 31(1), 2006, pp. 17-30. M. HUBERT, F. CHATEAURAYNAUD et J.-M. FOURNIAU, « Les chercheurs et la programmation de la recherche : du discours stratégique à la construction de sens » in Quaderni, $\mathrm{n}^{\circ} 77(1)$, 2012, pp. 85-96.

P. MILOT, « La reconfiguration des universités selon l'OCDE. Économie du savoir et politique de l'innovation ». In Actes de la recherche en sciences sociales, no 148 (3), pp.68-73.

J. NIOSI (éd.), New Technology Policy and so- cial Innovations in the Firm (2 ${ }^{\mathrm{e}}$ éd.), New York, Routledge, 1994.

OCDE, Principaux indicateurs de la science et de la technologie, 2014(1).

OCDE, Mesurer l'innovation. Un nouveau regard. Stratégie de l'OCDE pour l'innovation, 2010.

OCDE, Manuel d'Oslo. Principes directeurs pour le recueil et l'interprétation des données sur l'innovation. (3 éd), 2005.

OCDE, Société du savoir et gestion des connaissances. Enseignement et compétences, 2000.

J. OWEN-SMITH et W.W. POWELL, "Carrières et contradictions en sciences de la vie : réponses du corps académique aux transformations de la connaissance et de ses utilisations », in Sociologie du travail, $\mathrm{n}^{\circ} 46,2004$, pp. 347-377.

C. VILKAS, 'Des pairs aux experts : l'émergence d'un 'nouveau management' de la recherche scientifique ?', in Cahiers Internationaux de Sociologie, no 126 (1), 2009, pp. 61-79. 
$\mathrm{N} \cdot \mathrm{O} \cdot \mathrm{T} \cdot \mathrm{E} \cdot \mathrm{S}$

1. Disponible sur le site web d'Industrie Canada.

2. Environnement et agriculture, santé et sc. de la vie, ressources naturelles et énergie, TICs et fabrication de pointe.

3. Voir le site internet de la FCI à l'adresse www.innovation.ca pour plus d'informations sur l'institution, les projets qu'elle soutient et ses modes d'évaluation et de sélection.

4. Disponible sur le site web du Centre de services partagés du Québec.

5. Voir par exemple le mémoire soumis par l'Association francophone pour le savoir à cette adresse $: \underline{h t t p: / /}$ www.acfas.ca/publications/consultations/elaborationpolitique-nationale-recherche-innovation

6. Au Canada, le terme désignant le secteur disciplinaire des sciences pour l'ingénieur est 'génie', équivalent direct de 'engineeniring' (terme usuel dans toute l'Amérique).

7. Les phases doctorale et postdoctorale de l'enquête ont été réalisées avec l'appui financier du Conseil de recherches en sciences humaines du Canada et du Fonds Société et culture du Québec.

8. L'enquête initiale a été approuvée par le comité éthique de l'Université de Sherbrooke et sa suite répond à ses exigences et aux normes des organismes canadiens de financement de la recherche.

9. Incluant les universités, écoles, instituts et hôpitaux universitaires ou affiliés.

10. Les extraits ont été repérés et codés par catégorisation émergente et par raffinement (coding on). L'encodage a fait l'objet de tests d'accords interjuges qualitatifs et des matrices de croisement ont permis d'évaluer le poids relatif $( \pm)$ de ces thèmes (sans le quantifier systématiquement) en tenant compte du nombre de références et de cas ainsi que des liens entre les thèmes (overlaping codes). Les citations (originales ou traduites de l'anglais par l'auteure) ont été sélectionnées parce qu'elles sont très représentatives des énoncés encodés sous le même thème.

11 . Thèmes abordés par au moins $25 \%$ des répondants dans une arborescence de 257 codes (filtre : mot-clé 'Innovation' et mots de la même famille comme 'innover', 'innovant', etc.). Source : réponses à des questions ouvertes sur les éléments nuisibles et les améliorations/ catégorie : qualité de la recherche et de la formation à la recherche. $\mathrm{N}=$ nombre de répondants.

12. Le coût total est pris en compte en principe dans les nouvelles politiques scientifiques canadienne et québécoise mais il est trop tôt pour pouvoir discuter de la mise en œuvre de mesures associées. 


\section{$R \cdot E ́ \cdot S \cdot U \cdot M \cdot E ́$}

Cet article traite de la perception qu'ont les chercheurs de la notion d'innovation telle qu'elle est véhiculée dans les politiques scientifiques en sciences de la santé, sciences de la vie et génie. Réalisée à partir des données d'une enquête mixte et longitudinale amorcée en 2009 (832 participants), l'analyse rend compte d'interprétations contradictoires des termes et des objectifs des politiques ainsi que de leurs impacts parfois contreproductifs mettant en cause certaines pratiques dans le système universitaire canadien.

\section{Abstract \\ Innovation as perceived by Canadian researchers from so-called hard sciences.}

This article discusses researchers' perception of the notion of innovation as conveyed by science policy statements in Health Sciences, Life Sciences and Engineering. Based on data from a mixed longitudinal study initiated in 2009 (832 participants), the analysis reflects how researchers hold contradictory interpretations of the terms and objectives of the various policy documents. Our study also exposes some counterproductive impacts regarding different practices in the Canadian university system. 
